\title{
Exploiting Quench Dynamics in Spin Chains for Distant Entanglement and Quantum Communication
}

\author{
Hannu Wichterich and Sougato Bose \\ Dept. of Physics and Astronomy, University College London, \\ Gower Street, WC1E 6BT London, United Kingdom
}

(Dated: June 19, 2018)

\begin{abstract}
We suggest a method of entangling significantly the distant ends of a spin chain using minimal control. This entanglement between distant individual spins is brought about solely by exploiting the dynamics of an initial mixed state with Néel order if the lattice features nearest-neighbor XXZ interaction. There is no need to control single spins or to have engineered couplings or to pulse globally. The method only requires an initial non-adiabatic switch (a quench) between two Hamiltonians followed by evolution under the second Hamiltonian. The scheme is robust to randomness of the couplings as well as the finiteness of an appropriate quench and could potentially be implemented in various experimental setups, ranging from atoms in optical lattices to Josephson junction arrays.
\end{abstract}

The objective of quantum communication is to transfer a quantum state $|\phi\rangle$ from a sender (Alice) to a receiver (Bob) as accurately as possible. For this, Alice can simply encode $|\phi\rangle$ on a carrier and send it. Alternatively, she and Bob can use teleportation [1], for which they need to share a pair of particles in an entangled state $\left|\psi^{+}\right\rangle=\frac{1}{\sqrt{2}}(|\downarrow, \uparrow\rangle+|\uparrow, \downarrow\rangle) \cdot\left|\psi^{+}\right\rangle$, with appropriate operations and classical communications, enables the noiseless transmission of a state $|\phi\rangle$ from Alice to Bob. Photons are ideal for long distance quantum communication.

This paper is based on a manifestation of quantum communication which aims to connect distinct parts, i.e. registers, of a quantum computer. The idea is to use a many-body system (with permanent interactions) incorporating the sender, the receiver, and the mediating channel all together. All components are then stationary, and could be made of the same units. This setup does not require the interfacing of stationary qubits with photons. This could considerably reduce the complexity of interconnects between registers of a quantum computer which typically need to enable entanglement sharing over short distances i.e. several lattice sites. For the same reason, the physical movement of ions is considered seriously for communication between ion trap quantum registers [2]

Fig. 1 pictures our scenario, where Alice and Bob are situated at opposite ends of a one dimensional (1D) lattice of perpetually interacting spin- $\frac{1}{2}$ particles. We suggest a scheme which allows the establishment of a strong entanglement between Alice's and Bob's spins (the remotest spins of the lattice) without any requirement of local control for initializing the chain or for the subsequent dynamics. Neither the repeated switchings of any fields (local or global) nor any specially engineered couplings are required. This is close to the scenario of much work on state transfer through spin chains (e.g. Refs. $3,[4,[5,[6,7])$, but here the lattice interactions "generate" entanglement, as opposed to just "transferring" it. State transfer can itself be modified to yield entanglement generation schemes (eg. see [8]), but without a price (e.g. engineered couplings and local preparation),

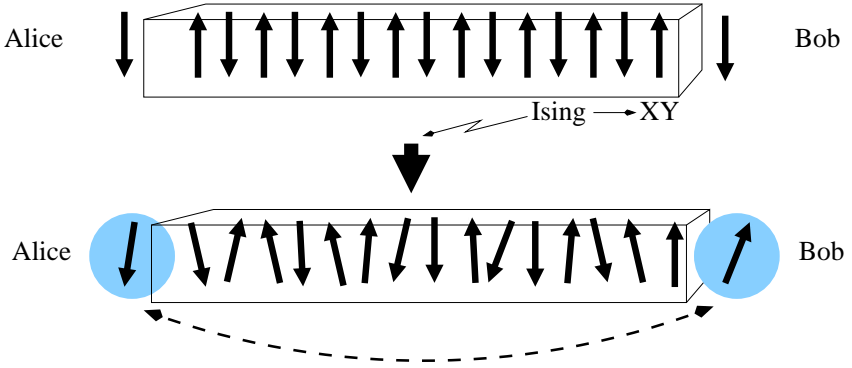

FIG. 1: (Color online) Schematic of our proposal of entangling distant spins. Alice and Bob are at opposite ends of the chain. First, the spin chain is initialized in the antiferromagnetic Ising groundstate. By a non-adiabatic switching to an $\mathrm{XY}$ interaction and subsequent time evolution quantum correlations (entanglement) is being established between Alice's and Bob's spins.

the amount of entanglement will be very small. Even without such price, our current mechanism provides a very high entanglement.

In our scheme, first the lattice of strongly interacting spins is cooled to its ground state. Then, upon instantly changing a global parameter in its Hamiltonian (i.e., performing a quench [11, 12, 13, 14, 15]), the pair of edge spins evolve to a highly entangled mixed state. This state is special in the sense that entanglement purification [16], a process that Alice and Bob can use to convert, by local actions, a few (say $n$ ) copies of the state to $m<n$ pure $\left|\psi^{+}\right\rangle$states, can be quite efficient. These $\left|\psi^{+}\right\rangle$could then be used to teleport any state from Alice to Bob. Though the scheme of this paper has a qualitative similarity with entangling the ends of a chain of uncoupled systems by a sudden switching of interactions [9, 10], it yields a much higher entanglement scaling better with the length of the chain. Also, as we will show, we actually start from a mixed initial state despite the cooling so the production of a high entanglement between ends is quite interesting.

Quenches have been actively studied in condensed mat- 
ter, usually in the thermodynamic limit [11] and were recently shown shown to make local subsystems relax towards maximum entropy 12]. In contrast, here we investigate whether a quench, when performed on a finite system, can produce a "long-range" entanglement between individual distant spins. Recently, the development of a qualitatively different form of entanglement, namely that between complementary blocks of a spin chain was shown to be possible after a quench [13, 14, 15]. This phenomenon was attributed to a heuristic picture in which several sources in a lattice emit oppositely moving pairs of entangled quasiparticles which reach distinct blocks to entangle them. However, a high entanglement between two individual spins is not guaranteed by the above picture - indeed for certain quenches in the quantum Ising model, where the above process entangles blocks [13], there is hardly any entanglement between the end spins. A more intricate process involving reflections of wavepackets at boundaries, and a cumulative contribution of the same entangled state from different sources to the end spins, can, for the quench we examine, create a very high entanglement between end spins at a special time. This is both directly relevant to quantum communications and measurable, as opposed to the entanglement of blocks.

Using a global quench to create entanglement at a distance is highly attractive because of the non-requirement of controlling single spins. Other low control ways of creating sizeable entanglement between distant spins include exploiting certain spin chains whose end spins are coupled weakly to the remaining ones [17, 19]. This is a rare engineered case, as generically the ground states of spin chain models are known to exhibit only very short ranged entanglement [18]. Another approach is based on localizable entanglement [20] where one entangles the end spins of a bulk system by individual measurements on the other spins, which can be challenging.

Returning now to the problem of entangling distant spins through a quench, consider a chain of $N$ spin- $\frac{1}{2}$ systems with nearest-neighbor XXZ interaction

$$
H=\sum_{k=1}^{N-1} \frac{J_{k}}{2}\left(\sigma_{k}^{\mathrm{x}} \sigma_{k+1}^{\mathrm{x}}+\sigma_{k}^{\mathrm{y}} \sigma_{k+1}^{\mathrm{y}}+\Delta \sigma_{k}^{\mathrm{z}} \sigma_{k+1}^{\mathrm{z}}\right)
$$

where the parameters $J_{k}$ and $\Delta$ denote the coupling strengths at bond $k$ and the anisotropy respectively, and $\sigma_{k}^{\mathrm{x}}, \sigma_{k}^{\mathrm{y}}$ and $\sigma_{k}^{\mathrm{z}}$ denote the Pauli operators acting on the spin at site $k$. We assume $J_{k}=J>0$ (homogeneous anisotropic coupling) in most parts of this work, unless we study disorder in later parts of this paper: To that end the couping is modulated as $J_{k}=J\left(1+\delta_{k}\right)$ with normally distributed random numbers $\delta_{k}$ with mean zero and standard deviation $\delta$. Eq. (11), corresponds to the Ising-Model for $\Delta \rightarrow \infty$, and the isotropic XY, or XX model for $\Delta=0$. As $\left[H, S_{\mathrm{z}}\right]=0$, with $S_{\mathrm{z}}=\sum_{k=1}^{N} \sigma_{k}^{\mathrm{z}}$, the total z-magnetization is a constant of motion.
We first formulate the analytic case of time-evolution of the Ising ground state, under action of the XX Hamiltonian. This corresponds to an instantaneous, i.e. idealized quench in the anisotropy parameter $\Delta_{1} \rightarrow \Delta_{2}$ with $\Delta_{1} \rightarrow \infty$ and $\Delta_{2}=0$ thereby crossing critical value $\Delta=1$, which separates the Néel-Ising-phase from the $\mathrm{XY}$-phase. Later on, in a purely numerical study we will allow for $1<\Delta_{1}<\infty$ and $0<\Delta_{2} \leq 1$. For $\Delta \gg J$ the Ising groundstate gets arbitrarily close to the ideal Néel state, which is twofold degenerate in the absence of an external field. These ideal Néel states arise from the perfectly polarized state $\left|\Downarrow_{N}\right\rangle$ upon flipping every other spin: $\left|\mathcal{N}_{1}\right\rangle \equiv\left|\downarrow_{1}, \uparrow_{2}, \downarrow_{3}, \cdots\right\rangle$ and $\left|\mathcal{N}_{2}\right\rangle \equiv\left|\uparrow_{1}, \downarrow_{2}, \uparrow_{3}, \cdots\right\rangle$. Note that these two states turn into each other by a spin flip at each place, i.e. $\left|\mathcal{N}_{1}\right\rangle=\left(\prod_{k=1}^{N} \sigma_{k}^{\mathrm{x}}\right)\left|\mathcal{N}_{2}\right\rangle$ and vice versa. In an experiment, the initial preparation of the Néel-Isinggroundstate, will yield, at low enough temperatures, an equal mixture of both Néel orders, and negligible admixture of higher energy eigenstates. We adopt the notion of thermal ground state from [18] for

$$
\rho_{0}=\frac{1}{2}\left(\left|\mathcal{N}_{1}\right\rangle\left\langle\mathcal{N}_{1}|+| \mathcal{N}_{2}\right\rangle\left\langle\mathcal{N}_{2}\right|\right)
$$

which exhibits the same symmetries as the Ising Hamiltonian $H(\Delta \rightarrow \infty)$, as opposed to each individual, degenerate ground state of the antiferromagnetic Ising-chain.

After diagonalization following a Jordan-Wigner transformation, we are provided the explicit time dependence of the local Fermi creation operators $c_{k}^{\dagger} \equiv$ $\left(\prod_{l=1}^{k-1}-\sigma_{l}^{\mathrm{z}}\right) \sigma_{k}^{+}$, with $\sigma_{k}^{+} \equiv \frac{1}{2}\left(\sigma_{k}^{\mathrm{x}}+i \sigma_{k}^{\mathrm{y}}\right)$, which reads

$$
\begin{aligned}
c_{k}^{\dagger}(t) & =\sum_{l=1}^{N} f_{k, l}(t) c_{l}^{\dagger} \\
f_{k, l}(t) & \equiv \frac{2}{N+1} \sum_{m=1}^{N} \sin \left(q_{m} k\right) \sin \left(q_{m} l\right) e^{-i E_{m} t},
\end{aligned}
$$

with $E_{m}=2 J \cos \left(q_{m}\right)$ and $q_{m}=\frac{\pi m}{N+1}$.

We now investigate the dynamics of the long range nonclassical correlations, i.e. the entanglement between the first and the last spin of the chain, provided the system is initially prepared in the global state (2) and assuming $\Delta=0$ for the subsequent time evolution. The state (reduced density operator) $\rho_{1, N}$ of spins at sites 1 and $N$ of the chain in the $\{|\uparrow \uparrow\rangle,|\uparrow \downarrow\rangle,|\downarrow \uparrow\rangle,|\downarrow \downarrow\rangle\}$ basis has only the following non-zero elements

$$
\begin{aligned}
& \left\langle\uparrow \uparrow\left|\rho_{1, N}\right| \uparrow \uparrow\right\rangle=\left\langle\downarrow \downarrow\left|\rho_{1, N}\right| \downarrow \downarrow\right\rangle=a,\left\langle\uparrow \downarrow\left|\rho_{1, N}\right| \uparrow \downarrow\right\rangle= \\
& \left\langle\downarrow \uparrow\left|\rho_{1, N}\right| \downarrow \uparrow\right\rangle=b,\left\langle\uparrow \downarrow\left|\rho_{1, N}\right| \downarrow \uparrow\right\rangle=\left\langle\downarrow \uparrow\left|\rho_{1, N}\right| \uparrow \downarrow\right\rangle=c,
\end{aligned}
$$

which are entirely expressible in terms of second moments of the Fermi operators (3). From $a=\left\langle\sigma_{1}^{+} \sigma_{1}^{-} \sigma_{N}^{+} \sigma_{N}^{-}\right\rangle$and $c=\left\langle\sigma_{1}^{-} \sigma_{N}^{+}\right\rangle$, where $\langle\cdots\rangle=\operatorname{Tr}\{\rho(t) \cdots\}$ and $\rho(t)=$ 


$$
\begin{gathered}
e^{-i H t} \rho_{0} e^{i H t} \text {, we find } \\
\qquad \begin{array}{c}
a=\left\langle c_{1}^{\dagger} c_{1}\right\rangle_{1}\left\langle c_{N}^{\dagger} c_{N}\right\rangle_{1}-\left\langle c_{1}^{\dagger} c_{N}\right\rangle_{1}\left\langle c_{N}^{\dagger} c_{1}\right\rangle_{1} \\
-\frac{1}{2}\left(\left\langle c_{1}^{\dagger} c_{1}\right\rangle_{1}+\left\langle c_{N}^{\dagger} c_{N}\right\rangle_{1}-1\right) \\
c=\frac{1}{2}\left((-1)^{M+1}\left\langle c_{N}^{\dagger} c_{1}\right\rangle_{1}+\text { c.c. }\right) .
\end{array}
\end{gathered}
$$

Here, $\langle\cdots\rangle_{1}$ denotes the expectation value in the Schrödinger picture with respect to the initial state $\left|\mathcal{N}_{1}\right\rangle$, which facilitates factoring higher moments according to Wick's theorem, and $M$ is the conserved number of spin up states in the dynamical state $\left|\mathcal{N}_{1}(t)\right\rangle$, i.e. $M=N / 2$ for even $N$ and $M=(N-1) / 2$ for odd $N$. The remaining element $b$ is determined by the trace constraint $2 a+2 b=1$. Analogous formulas for ground and thermal states of the XX spin chain were reported in [19]. The second moments occurring in (6) can be conveniently evaluated in the Heisenberg picture, where

$$
\left\langle c_{i}^{\dagger}(t) c_{j}(t)\right\rangle=\sum_{k, l=1}^{N} f_{i, k}(t) f_{j, l}^{*}(t)\left\langle c_{k}^{\dagger}(0) c_{l}(0)\right\rangle .
$$

As $\left\langle c_{k}^{\dagger}(0) c_{l}(0)\right\rangle_{1}=\delta_{k, l} \delta_{k, 2 m},(m=1,2, \ldots, M)$, we get

$$
c=\frac{1}{2}(-1)^{M+1} \sum_{m=1}^{M} f_{N, 2 m}(t) f_{1,2 m}^{*}(t)+c . c .
$$

This expression underlines that quantum correlations between the end spins are a superposition of contributions from spin flips originating from every other site $2 m$.

We now examine whether a given supply of systems, all described by the same mixed state $\rho_{1 N}$ from Eq. (5), can be converted to a smaller subset of $\left|\psi^{+}\right\rangle$states through local actions by Alice and Bob and classical messaging between them, for subsequent use in teleportation. A general criterion [16] for this procedure, called entanglement purification, to be possible for mixed state $\rho$ of two qubits is expressed in terms of the fully entangled fraction $f \equiv \max (\langle e|\rho| e\rangle)$. Here the maximum is taken with respect to all maximally entangled states $\{|e\rangle\}$. The criterion reads $f>\frac{1}{2}$, and is adapted to our particular problem as $\max (b+c, b-c)>\frac{1}{2}$ in view of Eq. (5) under the trace constraint $a+b=\frac{1}{2}$.

The function $f$ will feature a first maximum some time $T_{\max }$ after the instant of quench (Fig 2, left inset) and this is always found (by a numerical search for different $N$ ) to scale as $T_{\max } \sim \frac{N}{\pi J}$ (Fig. 2, right inset - in fact, $T_{\max }$ is slightly lower than $\frac{N}{\pi J}$ ). Interestingly, for chains with even $N$ and $\rho_{0}$ as the initial state, $\rho_{1, N}$ is always unentangled (separable). This is because $\left|\mathcal{N}_{1}\right\rangle$ contributes $|\xi\rangle=\alpha|\downarrow \uparrow\rangle+i \beta|\uparrow \downarrow\rangle$ to $\rho_{1, N}$ (with real $\alpha$ and $\beta$ ) while symmetry implies $\left|\mathcal{N}_{2}\right\rangle$ to contribute an equal amount of $|\tilde{\xi}\rangle=\sigma_{x} \otimes \sigma_{x}|\xi\rangle$ so that $|\xi\rangle\langle\xi|+| \tilde{\xi}\rangle\langle\tilde{\xi}|$ is separable. Therefore, in Fig. 2 and henceforth, we consider odd $N$.

A high entanglement specifically between the end spins requires the reflection of wavepackets at the boundaries,

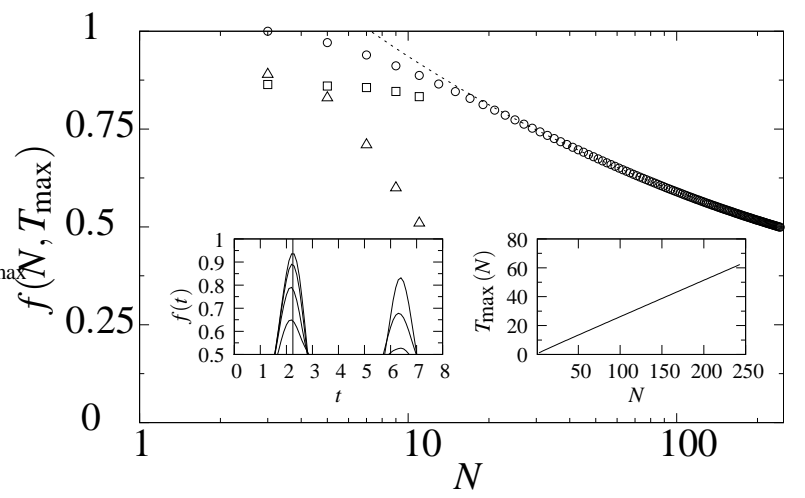

FIG. 2: Fully entangled fraction $f$ evaluated at time $t=T_{\max }$ as a function of system size $N$ in a semilogarithmic scale. The data points for the analytic quench scenario (circles) exceed 0.5 for chains up to $N=241$ spins. We find a good agreement of these data for $N \geq 25$ to a function of the form $g^{\text {fit }}(N) \propto 1.42(9) N^{-\nu}$ with $\nu=0.22(9)$ (dashed curve). For $N \leq 11$ the figure is supplemented with data from numerical diagonalization for more general quench characteristics $\left(\Delta_{1} \rightarrow \Delta_{2}\right):(\infty \rightarrow 1)$ (triangles) and $(3 \rightarrow 0)$ (squares) . Left inset: Typical time evolution of $f$ in a quench $(\infty \rightarrow 0)$ for $N=7$ with first maximum at $T_{\max }$ (vertical line). Disorder ( $\delta=0,0.1,0.2,0.3$ from top to bottom curves respectively) is seen not to affect the amount of entanglement seriously and leaves $T_{\max }$ almost unchanged (average taken over 100 realizations). Right inset : Linear scaling of $T_{\max }$ with $N$.

the initial Néel order and an independent propagation of different fermions after quench. Consider $\left|\mathcal{N}_{1}\right\rangle$ (with odd $N)$ to have its central spin $|\uparrow\rangle$. Each $|\uparrow\rangle$ of $\left|\mathcal{N}_{1}\right\rangle$ (or each $|\downarrow\rangle$ of $\left.\left|\mathcal{N}_{2}\right\rangle\right)$ is the location of a fermion which goes to a superposition of left and right moving wavepackets due to the dynamics. From Ref. 13, 14] we know that such dynamics alone suffices to entangle blocks, but entangling individual spins requires much more. $T_{\max }$ is close to the time it takes a fermion to traverse half the chain. At $T_{\max }$ a fermion initially, say, in the left half of the chain would have the peak of its left wavepacket reflected at the left end and traveled a distance $d$ from that end, while the peak of its right wavepacket, yet to encounter an end, would be about the same distance $d$ from the right end. This implies that for each fermion, the amplitudes of its presence at the two ends will be nearly equal at $T_{\max }$. $\Delta_{2} \sim 0$ (free model) can ensure an independent dynamics of each fermion. Both $\left|\mathcal{N}_{1}\right\rangle$ and $\left|\mathcal{N}_{2}\right\rangle$ ensure that each fermion crosses either an even or an odd number of others to reach either end, so that there is no net effect of exchange phases. Thus each fermion will independently contribute a state very close to $\left|\psi^{+}\right\rangle$to $\rho_{1, N}\left(T_{\max }\right)$ (the nearer to an end a fermion is initially, the more its contribution deviates from $\left.\left|\psi^{+}\right\rangle\right)$. The cumulative contribution of the same entangled state from all fermions at $T_{\max }$ creates a very high entanglement between 1 and $N$. If the different fermions contributed distinct entangled states (e.g. $|\downarrow, \uparrow\rangle+e^{i \phi}|\uparrow, \downarrow\rangle$ with different $\phi$ 's), the cumulative 
entanglement could have been vanishingly small even if block entanglement would be high.

We now provide an estimate for the supply of impure pairs required as input in order to produce one almost pure maximally entangled state, i.e. $f^{\text {out }} \geq 0.99$. At time $T_{\max }$, our mixed state becomes $\rho_{1, N} \simeq f\left|\psi^{+}\right\rangle\left\langle\psi^{+}\right|+$ $\frac{(1-f)}{2}(|\uparrow, \uparrow\rangle\langle\uparrow, \uparrow|+| \downarrow, \downarrow\rangle\langle\downarrow, \downarrow|)$, and the so called recurrence method of purification, described in detail in [16], simplifies decisively (error correction, as usable for communication through disordered chains 21], could also be used). Starting from an ensemble of impure pairs with individual $f=0.544$, which is the value for $N=151$ in Fig. 2, will require 5 iterations of the purification scheme on $\sim 361$ input pairs to achieve a single pair with $f^{\text {out }}=0.996$. In comparison, for a particular chain of $N=9$ spins, which is also a representative number for possible experiments, we have initially $f=0.9117$ and will need $\sim 3$ impure pairs to be purified into an almost perfect $\left|\psi^{+}\right\rangle$in a single iteration step until our threshold is exceeded by $f^{\text {out }}=0.991$. We have also studied numerically and plotted in Fig 2 more general quenches. The results suggest that rather than having a perfect Néel order in the initial state $\left(\Delta_{1} \rightarrow \infty\right)$, it is more vital to propagate according to the free fermion Hamiltonian $\left(\Delta_{2}=0\right)$ in order to achieve purifiable entanglement for larger $N$. Our scheme is also suprisingly robust towards the type of disorder under consideration, i.e. random couplings. As shown in the left inset of Fig, for random offsets as large as an average ten per cent of the coupling strength $J$ the amplitude of the fully entangled fraction at $T_{\max }$ as well as $T_{\max }$ itself remain virtually unaffected.

The verifiability of our results is within experimental reach. Promising realizations of $X X Z$ spin chains are Josephson junction arrays 22] (where one can tune $J$ and $\Delta$ by varying voltages [23] or magnetic flux [24]). Spin-spin interactions in optical lattices 25] have recently been demonstrated [26]. These experiments also provide a global method of preparing the initial state: pairwise spin triplets $\left|\psi^{+}\right\rangle$are first prepared in the double wells of a superlattice, a magnetic field gradient is then applied to attain a $|\uparrow, \downarrow\rangle$ state in each double well, and finally, the long lattice instantaneously ramped down to create a Néel state. Ensembles of finite lattices should be simulable with superlattices and measurements of spin states are possible [26]. We have ignored decoherence for the moment in view of the relevant realizations (optical lattices are an ideal arena to study quenches in absence of environments [27], and small $N$ Josephson junction arrays do not have significant decoherence over our $\left(T_{\max }<\frac{N}{J}\right)$ time-scales [24] $)$. Trapped ions are also known to simulate spin chain Hamiltonians and their non-adiabatic changes 28]. In future, it would be interesting to study the influence of a finite quench rate on the entanglement between the end spins. We started from a Hamiltonian whose eigenstates are product (un- entangled) states when written in terms of a local basis i.e., the spin states at given sites of a lattice. We then quenched to a nearly free Hamiltonian which has momenta-like eigenstates. Thus a similar behavior may show in other models, e.g. Hubbard Hamiltonians when one quenches from an appropriate product of localized states to a free (such as the superfluid) parameter regime. Subsequent to the the work reported here, similar entanglement dynamics has also been found by other authors 30] in driven ferromagnetic chains, which maps to our case in certain limits.

Summary:- Non-equilibrium dynamics of a spin chain after a quench [11, 12, 13, 14, 15] is an area of intense activity. The scaling of distant spin-spin correlations after quenches is topical 31, 32 with interesting instances of polynomial scaling [31]. Much harder is to make the truly "quantum" correlations (or entanglement) between individual distant spins "substantial" and scale polynomially, which we have shown to be possible through a certain global quench. This is important because the entanglement between individual spins in complex systems, a quantity of wide interest [29], is easily measurable through correlation functions, but is notoriously short ranged [18]. Our scaling of entanglement $\left(\sim N^{-0.22}\right)$ seems significantly superior to that in the statics or dynamics of any spin model studied so far (except for specialized couplings [17]). Additionally, that we start from a mixed state and obtain so much entanglement between distant spins that it is efficiently purifiable to $\left|\psi^{+}\right\rangle$, at least for small $(N \sim 9)$ chains, and usable for linking solid-state based quantum registers is interesting. Tests of our results, e.g., in finite chains in optical superlattices [26], seem feasible.

The studentship of HW is supported by the EPSRC, UK. SB acknowledges EPSRC grant EP/D073421/1, the QIPIRC, the Royal Society and the Wolfson Foundation. We thank T. Boness, P.Sodano, A. Kay and particularly M. Cramer for very helpful comments.

[1] C. H. Bennett et al., Phys. Rev. Lett. 70, 1895 (1993).

[2] D. Kielpinski, C. Monroe, and D. J. Wineland, Nature 417, 709 (2002).

[3] S. Bose, Phys. Rev. Lett. 91, 207901 (2003).

[4] T. J. Osborne and N. Linden, Phys. Rev. A 69, 052315 (2004).

[5] M. Christandl, N. Datta, A. Ekert, and A. J. Landahl, Phys. Rev. Lett. 92, 187902 (2004); C. Di Franco, M. Paternostro, and M. S. Kim, Phys. Rev. Lett. 101, 230502 (2008).

[6] J. Fitzsimons and J. Twamley, Phys. Rev. Lett. 97, 090502 (2006); G. A. Paz-Silva, S. Rebic, J. Twamley and T. Duty, Phys. Rev. Lett. 102, 020503 (2009).

[7] V. Giovannetti and D. Burgarth, Phys. Rev. Lett. 96, 030501 (2006).

[8] I. D'Amico, B. W. Lovett and T. P. Spiller, Phys. Rev. 
A 76, 030302(R) (2007).

[9] J. Eisert, M.B. Plenio, S. Bose and J. Hartley, Phys. Rev. Lett. 93, 190402 (2004).

[10] D.I. Tsomokos, M.J. Hartmann, S.F. Huelga and M.B. Plenio New Journal of Physics 9, 79 (2007).

[11] K. Sengupta, S. Powell, and S. Sachdev, Phys. Rev. A 69, 053616 (2004).

[12] M. Cramer, C. M. Dawson, J. Eisert, and T. J. Osborne, Phys. Rev. Lett. 100, 030602 (2008).

[13] P. Calabrese and J. Cardy, J. Stat. Mech. P04010 (2005).

[14] G. De Chiara, S. Montangero, P. Calabrese, and R. Fazio, J. Stat. Mech. P03001 (2006).

[15] L. Cincio, J. Dziarmaga, M. M. Rams, and W. H. Zurek, Phys. Rev. A 75, 052321 (2007).

[16] C. H. Bennett, D. P. DiVincenzo, J. A. Smolin, and W. K. Wootters, Phys. Rev. A 54, 3824 (1996).

[17] L. Campos Venuti, C. Degli Esposti Boschi, and M. Roncaglia, Phys. Rev. Lett. 96, 247206 (2006).

[18] A. Osterloh, L. Amico, G. Falci, and R. Fazio, Nature 416, 608 (2002); T. J. Osborne and M. A. Nielsen, Phys. Rev. A 66, 032110 (2002).

[19] L. Campos Venuti, S. M. Giampaolo, F. Illuminati, and P. Zanardi, Phys. Rev. A 76, 052328 (2007)
[20] F. Verstraete, M. Popp, and J. I. Cirac, Phys. Rev. Lett. 92, 027901 (2004).

[21] J. Allcock and N. Linden, Phys. Rev. Lett. 102, 110501 (2009).

[22] R. Fazio and H. van der Zant, Phys. Rep. 355, 235 (2001).

[23] D. Giuliano and P. Sodano, Nucl. Phys. B 711, 480 (2005).

[24] A. Lyakhov and C. Bruder, New J. Phys. 7, 181 (2005).

[25] L.-M. Duan, E. Demler and M. D. Lukin, Phys. Rev. Lett. 91, 090402 (2003).

[26] S. Trotzky et al., Science 319, 295 (2008).

[27] M. Cramer et. al., Phys. Rev. Lett. 101, 063001 (2008).

[28] D. Porras and J. I. Cirac, Phys. Rev. Lett 92, 207901 (2004).

[29] A. Osterloh, L. Amico, R. Fazio and V. Vedral, Rev. Mod. Phys. 80, 517 (2008).

[30] F. Galve et. al., Phys. Rev. A 79, 032332 (2009).

[31] F. Igloi and H. Rieger, Phys. Rev. Lett 85, 3233 (2000).

[32] P. Calabrese and J. Cardy, Phys. Rev. Lett. 96, 136801 (2006). 